RESEARCH REPORT

\title{
EFFECTIVENESS OF VIRTUAL REALTY IN RECOVERY OF MOTOR FUNCTION IN POST-STROKE HEMIPARESIS: A QUASI-EXPERIMENTAL STUDY
}

\begin{abstract}
Virtual Reality(VR) is a novel treatment approach for stroke rehabilitation as it enables individuals to reproduce standard controlled movements to accomplish functional task. In contrast to conventional rehabilitation that is monotonous, long-term and challenging, VR increases patient interest in rehabilitation thereby reduces the boredom and adaptation therefore enhances the visuo-spatial feedback. It has been observed that approximately $65 \%$ of the stroke survivors suffer hemiparesis that predominantly leads difficulty in performing daily living activities. Therefore, this study aims to assess the sensorimotor and functional performance in post-stroke hemiparetic patients using VR based gaming system. A total of 81 participants who had first-ever ischemic or hemorrhagic stroke with onset of $>6$ months, enrolled in post-acute rehabilitation program at Tertiary Care Hospital were included. The VR gaming software named Wonder Tree (WT) $\odot$ was used for the intervention. All participants were required to play two different games i.e. bucket ball and bubble pop for 15-20 minutes, 3 days/week for the duration of 8th weeks. Participants were assessed on baseline and post-intervention on Fugyl-Meyer Assessment for Upper Extremity (FMA-UE) and Wolf Motor Function Test (W.MFT) to assess sensorimotor function and functional skills. Participants showed significant improvement in sensorimotor function and functional abilities $(p<0.05)$ after 8 th weeks of intervention. It was concluded that VR gaming is effective in improving motor recovery in post-stroke hemiparesis. Besides, future clinical trials are needed to be conduct to establish VR gaming system as an alternative to conventional stroke rehabilitation.
\end{abstract}

\section{KEYWORDS}

Virtual Reality, Stroke, Upper Extremity, Paresis, Neuroplasticity, Rehabilitation, Range of Motion

\author{
Bakhtawar Saleem \\ Occupational Therapist \\ Ziauddin Hospital \\ bakhtawar.saleem@gmail.com
}

[Saleem B. Effectiveness of Virtual Reality in Recovery of Motor Function in Post-Stroke Hemiparesis: A Quasi-Experimental Study. Pak. j. rehabil. 2018;7(2):45-51] 


\section{INTRODUCTION}

Cerebrovascular Accident (CVA), referred to as stroke is the leading cause of death and long-term disability in adults'. The annual stroke incidence based on crude and sex-adjusted age is $95 / 100,000$ persons per year in the tenure 2000-2016². In Pakistan, the mean age affected by stroke is 59 years that is approximately 10 years earlier as compared to Western countries ${ }^{2}$. In particular, substantial portion of post-survivors typically experiences neurological sequelae or prolonged disease-related complications that may have adverse effects on patient's quality of life ${ }^{3-6}$. Therefore, the foremost aim of stroke rehabilitation emphasizes on improving functional independence and maximizes quality of life, ultimately reduces the level of dependen$c y^{3}$. However, true incidence of stroke is still unknown due to lack of multi-center epidemiological studies. It has been observed that approximately $65 \%$ of the stroke survivors suffer hemiparesis ${ }^{7}$, a second-most frequently recognized impairments following stroke causes either complete or incomplete paralysis affecting either of the body side ${ }^{8}$. This condition predominantly leads difficulty in performing daily living activities. However, the course of functional recovery in post-stroke hemiparetic patients may vary among individuals with respect to right or left hemiparesis-10. From the last three decades, the field of stroke rehabilitation has recent advances constitutes of Non-Invasive Brain Stimulation, Constraint Induced Movement Therapy (CIMT), Robotics and Virtual Reality (VR) with an emphasis to maximize their mobility, decrease disability, community integration, subsequently reduces the disease burden and better quality of life" ${ }^{1}$. At present, VR is a novel treatment approach, defined as the computer-generated interactive simulated environment that allows users to engage with real-world objects or events with an aptitude to provide repetitive and task-specific training. The system included multisensory provision constitutes of visual, auditory and tactile feedback that is easy to be implemented hence the program has potential to optimize participant performance, motivation and interest in rehabilitation ${ }^{12}$.

Moreover, VR is found to be well-suited for stroke rehabilitation as the treatment provides concurrent feedback with respect to patient's ability ${ }^{13}$. Exercise in simulated VR environment enables individuals to reproduce standard movements with control in order to accomplish functional task that can be adequately manipulated with respect to individual's treatment ${ }^{14}$. In contrast to conventional rehabilitation which is monotonous, long-term, least interesting and adaptable whereas VR increases patient interest in rehabilitation which results in the reduction of boredom and adaptation, also enhances the visuo-spatial feedback ${ }^{15}$. Furthermore, to the best of author's knowledge, no studies have been conducted to determine the exercise dosage in recovery of post-stroke impairments ${ }^{16}$.
Choi et $\mathrm{al}^{17}$ demonstrated that commercial VR gaming is effective in recovery of gross motor functions and improving daily living activities in post-stroke survivors with mild-to-severe impairments. Additionally, Commercial VR gaming would be an effective therapeutic alternative as it provides repetitive and task-specific training in stroke rehabilitation. However, the system usage has been limited only to the patients with moderate grip strength and flexibility. Unfortunately, most of the VR systems and equipment are expensive and requires adequate space therefore the intervention can only be administered in hospital-based or clinical setting. On the contrary, new style of commercial VR technologies such as Wii or Nintendo is relatively inexpensive, easy to use and reported to promote functional recovery following stroke. At present, commercial VR gaming are reported to be used by $1 / 5$ th of therapist in stroke rehabilitation while 3/5th use available VR games, yet the intervention should only be commenced with recommended dosage and use18. Moreover, VR environment provides opportunity to make treatment enjoyable; despite of the fact some therapists also described barriers related to time duration, space and treatment $\operatorname{costs}^{18}$. Precisely, several features of VR gaming may be relevant in neurorehabilitation according to its complexity and intensity that may more effectively stimulates cortical reorganization, making sessions more pleasant. In this context, participants are continuously challenged by newly designed tasks, that implies a more active participation in exercise program require to hastening the recovery process, potentially improving the rehabilitation outcomes ${ }^{19}$.

Furthermore, some studies also indicated effectiveness of VR in combination to conventional rehabilitation for functional recovery of post-stroke deficits of upper extremity. Similarly, a study conducted by da Silva et $a^{20}$ used VR gaming program in addition to conventional rehabilitation indicated that VR group showed significantly improved motor recovery, speed and better scores on assessment parameters hence indicated VR as a promising tool for neurorehabilitation. Nevertheless, more empirical evidences of VR based standardized protocol are needed to establish to target specific impairments of upper extremity prompting functional recovery with respect to heterogeneity of population 16 . Despite of the presence of intensive rehabilitation of stroke, limited data is available regarding functional outcomes in unilateral or bilateral hemiparetic patients9. Likewise, Sapnosiket $\mathrm{al}^{21}$ determined positive results of VR in improving upper limb motor function. Though, author also highlighted disparity in outcome measures due to limited studies that may lead to study bias. More VR trials are needed to be addressed to assess cognition, executive function, visuo-spatial and ADL's performance.

Consecutively, as per the reported statistics, the stroke pattern in Asia demonstrates geographical diversity. As the burden of disease is rising, associat- 
ed conditions are evolving with time. Therefore, substantial measures are needed to be addressed in order to prevent stroke related complications that may leads to morbidity or mortality². Moreover, large-scale epidemiological studies should be conducted to observe disease related consequences in order to plan interventions to reduce disease burden. Therefore, the purpose of our study is to investigate the effectiveness of commercial VR based gaming system in improving sensorimotor and functional performance of hemiparetic patients. This study will also serves as a potential evidence for future clinical trials.

\section{METHODOLOGY}

This Quasi-experimental study was conducted in Physiotherapy Department of a Tertiary Care Hospital in Karachi. Participants who were enrolled in post-acute care rehabilitation program were recruited using non-probability convenient sampling technique in the tenure of January 2017-Decemeber 2018. A total of 81 participants were included based on the following inclusion and exclusion criteria:

\section{Inclusion Criteria ${ }^{22-23}$}

- Both male and female patients aged 18-80 years who had first-ever ischemic or hemorrhagic stroke with onset of $>6$ months.

- Modified Ashworth Scale (MAS) scores <3.

- Mini-Mental Scale Examination (MMSE) scores >25.

- Upper extremity paresis with no loss of proprioception in fingers of affected side.

- Ability to raise paretic hand to the height of the nipple and voluntarily flex the paretic fingers.

- Participants willing to participate.

\section{Exclusion Criteria ${ }^{22-23}$}

- Patients with previous history of stroke.

- Reduced visual fields on both or single eye.

- Stroke patients with onset of < 6 months enrolled in special rehabilitation for upper extremity paresis such as CIMT, Botulinum Toxin (BTX) injections treatment etc.

- Serious medical conditions including uncontrolled hypertension, acute pulmonary heart diseases, renal dysfunctions, severe cognitive impairment or psychiatric disorders.

\section{Data Collection Tool ${ }^{24-25}$}

Two assessment parameters were included for the assessment of motor function of UE:

- Fugyl-Meyer Assessment Scale for Upper Extremity (FMA-UE) is a stroke-specific scale to assess sensorimotor function, balance, joint pain and range of motion. The scale has an excellent reliability (alpha $=0.94$ to 0.98) and correlation ( $r=0.93$ ) for post-stroke hemi-paretic patients applied to determine stroke severity and motor recovery for further planning and treatment assessment.

- Wolf Motor Function Test (WMFT) is a clinical assessment tool to quantify upper limb functional abilities through timed-based functional tasks. The test has an excellent reliability (ICC=0.94) and validity ( $r=$ 0.86) forhemiparetic stroke patients.

\section{Intervention Protocol}

The protocol was based on Virtual Reality Gaming Software named Wonder Tree (WT) ${ }^{\odot}$. Before starting the intervention, all participants were initially assessed at the baseline on FMA-UE and WMFT. Afterwards, subjects were given an average of three trials to provide clear understanding of the games and playing device. Two different games i.e. bucket ball and bubble pop were selected for the trials that were required to be played for 15-20 minutes, 3 days/week for the duration of 8th weeks. Both the games emphasized on the use of affected and sound upper extremities for the motor function recovery. Post-assessment was performed at 8th week after the end of trial. Detailed gaming protocol is represented in Table-1.

\section{Data Analysis}

Data was analyzed on IBM SPSS (Statistical Package

\begin{tabular}{|c|c|c|}
\hline \multicolumn{3}{|c|}{ Table 1. Wonder Tree Gaming Protocol ${ }^{26}$} \\
\hline Game & Aim & Procedure \\
\hline Bucket Ball & $\begin{array}{l}\text { Aim This game is designed to improve } \\
\text { bi- lateral upper extremity coordination, } \\
\text { attention span, visuo-spatial skills } \\
\text { and decision making skills. }\end{array}$ & $\begin{array}{c}\text { In an augmented 3D virtual environment, } \\
\text { participant is required to collect the balls and } \\
\text { put in to the bucket using bilateral arms for } \\
15-20 \text { minutes, 3days/week for the } \\
\text { duration of } 8 \text { th weeks. Scores was } \\
\text { noted after } 3 \text { trials. }\end{array}$ \\
\hline Bubble Pop & $\begin{array}{l}\text { The game helps to improve and-eye } \\
\text { coordination as well as sorting } \\
\text { skills and attention span. }\end{array}$ & $\begin{array}{l}\text { Similarly, in 3D environment, patient was asked to } \\
\text { pop the virtual bubbles by pointing finger using } \\
\text { hand-eye coordination of affected upper extremity } \\
\text { for the duration of } 15-20 \text { minutes, } 3 \text { days/week for } \\
\text { total 8th weeks. The scores were observed after } 3 \text { trials. }\end{array}$ \\
\hline
\end{tabular}


for Social Sciences) Software version 20. Demographic characteristics of participants were presented in frequency, mean and standard deviations. Paired t-test was applied for within the group analysis on the basis of symmetrical normality assumption while $\mathrm{p}$-value <0.05 was considered significant.

\section{Ethical Considerations}

Subjects' data and related information was kept confidential. Before the commencement of intervention, participants were provided with participant information sheet tonsure about the details of the study in order to obtain the consent. The data was collected in the Institute/hospital after the permission from the Head of Department of Physiotherapy.

\section{RESULT}

A total of 81 participants included in the study consisted of 54 males and 27 females with mean age of $52.7 \pm 18.3$. Majority of the cases were presented with left sided stroke $(59.3 \%)$ whereas right sided stroke cases $(40.7 \%)$ were in minority based on ischemic and hemorrhagic sub-types respectively.

After 8th weeks post-intervention, participants showed significant improvement in sensorimotor function $(p<0.05)$ on FMA-UE scale with mean of $37.7 \pm 9.7$ from 30.4 \pm 8.7 . Similar significant improvement in functional abilities $(p<0.05)$ was observed on WMFT from $19.8 \pm 6.3$ to $22.9 \pm 7.0$ respectively as shown in Table-2.

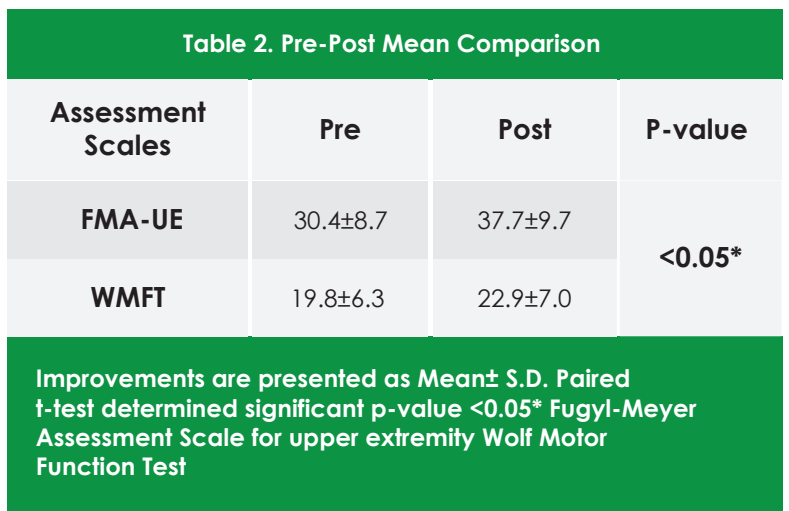

Moreover, the significant improvements were also observed on FMA-UE components in which motor function of upper extremity was significantly increased with mean of 26.2 \pm 5.0 . Further improvements were noted in wrist and function with mean values of $6.7 \pm 1.2$ and $3.8 \pm 2.3$ respectively. However, moderate significant improvement was evaluated in coordination and speed of participants with mean value of $3.2 \pm 1.8$. Besides, the results were significant for all FMA-UE components which showed improved recovery of motor function post-intervention as shown in Figure-1.

\section{Component of FMA-UE}

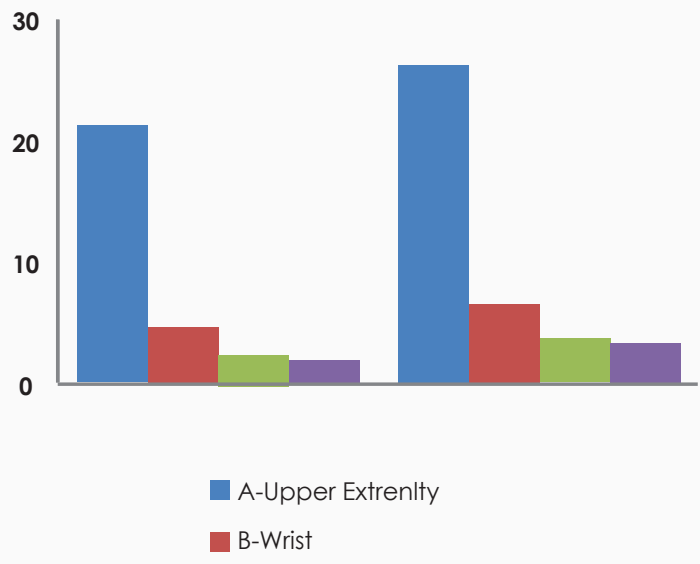

Figure 1. Represents prepost mean differences of FMA-UE sub-components

\section{DISCUSSION}

The outcomes of the study showed significant improvement in motor function recovery at 8th weeks of intervention. This proved the efficacy of VR based gaming to enhance motor and functional abilities following stroke subsequently increased quality of life of hemiparetic patients. Quite a few previous studies indicated that VR based intervention are potential to induce the mechanism of neuroplasticity in stroke survivors by activation of motor cortex and increased neuronal firing in stroke survivors ${ }^{27-35}$. Our results were consistent with the study of Soareset al ${ }^{36}$ who analyzed the therapeutic effects of VR based gaming in recovery of hemiparetic

patients. Results of the study indicated significant gains in FMA-UE with $34.0 \%$ increased range of motion abduction and $9 \%$ flexion with an increased quality of life. Although, these results were preliminary hence it was indicated that more empirical evidences are required to establish the effectiveness of VR based gaming in post-stroke rehabilitation. On the contrary, study conducted by da Silva Ribeiro ${ }^{37}$ demonstrated effectiveness of VR in combination with conventional physical therapy using Nintendo Wii in improving passive movements and decreased pain among hemiparetic stroke survivors. This showed that VR based intervention decreases disease-related immobility and disuse extremities which limits joint amplitude and exacerbates pain. Furthermore, Mouawadet $\mathrm{al}^{38}$ demonstrated Nintendo Wii as a rehabilitative tool for therapeutic reinforcement in home-based rehabilitation with an emphasis on passive and active upper extremities range of motion whereas a mean performance in time duration was also observed on 
WMFT. Similarly, our study also reported decreased mean for timed-functional tasks on WMFT. Though, certain studies reported disparity of outcome measures in stroke measures ${ }^{39-40}$. In this regard, our study focused on two different outcome measures with respect to specific function. In particular several researchers emphasized on commercial VR gaming software for clinical and home program practice whereas our study used an augmented VR system that is only affordable by the tertiary care units hence it may not use as home based rehabilitation. Likewise, a trial conducted by Turollaet a ${ }^{41}$ revealed that VR combined with conventional upper limb rehabilitation led significantly greater improvements at FMA-UE scores in comparison to conventional therapy only. Though, the study has an un-matched intervention that leads to difficulty in analysis of intervention and control group. Despite of the fact, our study contributes to establish VR based gaming as a sole intervention in improving sensorimotor function and timed performance of hemiparetic stroke patients. Although, some participants faced technological limitations due to general lack of awareness about gaming and knowledge of technological integration ${ }^{42}$. Furthermore, it is advocated that low-cost VR technologies should be used for clinical and home-based rehabilitation. Yet, in comparison to VR, conventional rehabilitation is reported to have the modest effect i.e. tedious for stroke survivors that lead to limited adherence and compliance to the treatment as well as high cost of rehabilitation ${ }^{39-44}$. In addition, we believe that our findings establish VR system as a valuable tool for recovery in stroke patients which allows them to engage in safe simulated environment where sensorimotor contingencies can be modulated on the valid principles of neurorehabilitation ${ }^{45}$. Although, multiple studies specifically endorsed VR gaming for motor recovery of upper extremity while some studies included more general outcomes post-stroke such as balance, gait or cognitive-motor training. Despite of the heterogeneity in study design, observed effectiveness of VR systems, target population and multiple outcome measures may constitute limitations in drawing valid conclusions. Unfortunately, some studies concluded that whether VR gaming is effective to improve motor function for activities of daily living remains unclear. Consequently, properly designed multicenter randomized controlled trials are needed to be established to reduce bias and to determine the efficiency and safety of VR gaming systems as an alternative therapeutic intervention to conventional rehabilitation in stroke patients ${ }^{39}$. In addition, it is also recommended to conduct the VR clinical trials at multiple sites with respect to different socioeconomic status to generalize the results in population. Therefore, further clinical trials are recommended to evaluate effectiveness of VR based intervention on principles of neuro rehabilitation comprises of repetitive task practice, implicit and explicit feedback in order to promote the recovery of paretic arm ${ }^{45}$.

\section{CONCLUSION}

VR gaming is effective in improving sensorimotor function and functional ability in post-stroke hemiparesis. Moreover, moderate to significant improvement was observed in FMA-UE components i.e. upper extremity, hand, wrist and coordination/speed. Besides, future clinical trials are required for establishment of VR gaming system as an alternative to conventional stroke rehabilitation.

\section{REFRENCES}

[1] Ma VY, Chan L, Carruthers KJ. Incidence, prevalence, costs, and impact on disability of common conditions requiring rehabilitation in the United States: stroke, spinal cord injury, traumatic brain injury, multiple sclerosis, osteoarthritis, rheumatoid arthritis, limb loss, and back pain. Arch Phys Med Rehabil. 2014;95(5):986-95.

[2] Mehndiratta MM, Khan M, Mehndiratta $P$, Wasay M. Stroke in Asia: geographical variations and temporal trends. J NeurolNeurosurg Psychiatry. 2014 85(12):1308-12.

[3] Gialanella B, Santoro R, Ferlucci C. Predicting outcome after stroke: the role of basic activities of daily living. Eur J PhysRehabil Med. 2012;48:1-9.

[4] Safer VB, Koseoglu BF. Timing of inpatient rehabilitation initiation in stroke patients: factors influencing early admission. J PhysTher Sci. 2015;27(6):1913-7.

[5] Takemasa S, Nakagoshi R, Murakami M, Uesugi $M$, Inove $Y$, Gotou $M$, Koeda $H$, Naruse $S$. Factors affecting quality of life of the homebound elderly hemiparetic stroke patients. J PhysTher Sci. 2014;26(2):301.

[6] Shinohara T, Usuda S. Are contents of physical therapy in nine Japanese hospitals for inpatients with stroke related to inpatients' and physical therapists' characteristics?.J PhysTher Sci. 2013;25(5):641-7.

[7] Go AS, Mozaffarian D, Roger VL, Benjamin EJ, Berry JD, Borden WB, Bravata DM, Dai S, Ford ES, Fox CS, Franco S. Executive summary: heart disease and stroke statistics-2013 update: a report from the American Heart Association. Circulation. 2013;127(1):143-52.

[8] Wist S, Clivaz J, Sattelmayer M. Muscle strengthening for hemiparesis after stroke: A meta-analysis. Ann PhysRehabil Med. 2016:59(2):114-24.

[9] Bindawas SM, Mawajdeh HM, Vennu VS, Alhaidary HM. Functional recovery differences after stroke rehabilitation in patients with uni-or bilateral hemiparesis. J Neuros- 
ci. $2017 ; 22(3): 186$.

[10] Schaefer SY, Mutha PK, Haaland KY, Sainburg RL. Hemispheric specialization for movement control produces dissociable differences in online corrections after stroke. Cerebral cortex. 2012;22(6):1407-19.

[11] AzamRathore F, Waheed A. Stroke rehabilitation in Pakistan: what we know and what we need to do?. PJNS. 2014;9(4):3-4.

[12] Hondori HM, Khademi M, McKenzie A, Dodakian L, Lopes CV, Cramer SC. Abstract T MP43: utility of augmented reality in relation to virtual reality in stroke rehabilitation. Stroke. 2014;45(suppl_1):ATMP43.

[13] Yamato TP, Pompeu JE, Pompeu SM, Hassett L. Virtual reality for stroke rehabilitation. PhysTher. 2016:96(10):1508-13.

[14] Soares AV, Woellner SS, Andrade CD, Mesadri TJ, Bruckheimer AD, Hounsell MD. The use of Virtual Reality for upper limb rehabilitation of hemiparetic Stroke patients. FisioterapiaemMovimento. 2014:27(3):309-17.

[15] Teasell R, Meyer MJ, McClure A, Pan C, Murie-Fernandez M, Foley N, Salter K. Stroke rehabilitation: an international perspective. Topics in stroke rehabilitation. 2009;16(1):44-56.

[16] Stinear CM. Prediction of motor recovery after stroke: advances in biomarkers. Lancet Neurol. 2017;16(10):826-36.

[17] Choi JH, Han EY, Kim BR, Kim SM, Im SH, Lee SY, Hyun CW. Effectiveness of commercial gaming-based virtual reality movement therapy on functional recovery of upper extremity in subacute stroke patients. Ann PhysRehabilMed.. 2014;38(4):485.

[18] Thomson K, Pollock A, Bugge C, Brady MC. Commercial gaming devices for stroke upper limb rehabilitation: a survey of current practice. DisabilRehabil Assist Technol. 2016;1 1 (6):454-61.

[19] Turolla A, Dam M, Ventura L, Tonin P, Agostini $M$, Zucconi C, Kiper P, Cagnin A, Piron L. Virtual reality for the rehabilitation of the upper limb motor function after stroke: a prospective controlled trial. J NeuroengRehabil 2013:10(1):85.

[20] da Silva Cameirão M, Bermudez i Badia S, Duarte 45E, Verschure PF. Virtual reality based rehabilitation speeds up functional recovery of the upper extremities after stroke: a randomized controlled pilot study in the acute phase of stroke using the rehabilitation gaming system. RestorNeurolNeurosci. 2011;29(5):287-98.

[21] Saposnik G, Mamdani M, Bayley M, Thorpe KE, Hall J, Cohen LG, Teasell R. Effectiveness of Virtual Reality Exercises in STroke Rehabilitation (EVREST): rationale, design, and protocol of a pilot randomized clinical trial assessing the Wii gaming system. Int J Stroke. 2010;5(1):47-51.

[22] Mizuno K, Abe T, Ushiba J, Kawakami M, Ohwa T, Hagimura K, Ogura M, Okuyama K, Fujiwara T,
Liu M. Evaluating the Effectiveness and Safety of the Electroencephalogram-Based Brain-Machine Interface Rehabilitation System for Patients With Severe Hemiparetic Stroke: Protocol for a Randomized Controlled Trial (BEST-BRAIN Trial). JMIR research protocols. 2018;7(12):e12339.

[23] Effect of Immersive Virtual Reality Usage on Upper Extremity Function in Stroke Patients: A Prospective, Double Blinded, Randomized Controlled Clinical Trial - ICH GCP - Clinical Trials Registry [Internet]. Ichgcp.net. 2018 [cited 25 April 2019]. Available from: https://ichgcp.net/clinical-trials-registry/NCT03135418.

[24] Fugl-Meyer Assessment of Sensorimotor Recovery After Stroke (FMA) - Stroke Engine [Internet]. Stroke Engine. 2019 [cited 25 April 2019]. Available from: https://www.strokengine.ca/en/assess/fma/.

[25] Psychometric Properties - Stroke Engine [Internet]. Stroke Engine. 2019 [cited 25 April 2019]. Available from: https://www.strokengine.ca/en/psycho/wmft_psycho/.

[26] WonderTree [Internet]. Wondertree.co. 2019 [cited 25 April 2019]. Available from: https://www.wondertree.co/wondergames/.

[27] Kalra L, Ratan R. Recent advances in stroke rehabilitation 2006. Stroke. 2007 Feb 1;38(2):235-7.

[28] Lucca LF. Virtual reality and motor rehabilitation of the upper limb after stroke: a generation of progress?. J Rehabil Med. 2009 ;41 (12):1003-6.

[29] Burke JW, McNeill MD, Charles DK, Morrow PJ, Crosbie JH, McDonough SM. Optimising engagement for stroke rehabilitation using serious games. The Visual Computer. 2009:25(12):1085.

[30] Jang SH, You SH, Hallett M, Cho YW, Park CM, Cho SH, Lee HY, Kim TH. Cortical reorganization and associated functional motor recovery after virtual reality in patients with chronic stroke: an experimenter-blind preliminary study. Arch Phys Med Rehabil. 2005;86(1 1):2218-23

[31] You SH, Jang SH, Kim YH, Hallett M, Ahn SH, Kwon YH, Kim JH, Lee MY. Virtual reality-induced cortical reorganization and associated locomotor recovery in chronic stroke: an experimenter-blind randomized study. Stroke. 2005:36(6):1 166-71.

[32] Teasell RW, Kalra L. What's new in stroke rehabilitation. Stroke. 2004;35(2):383-5.

[33] Merians AS, Jack D, Boian R, Tremaine $M$, Burdea GC, Adamovich SV, Recce M, Poizner $H$. Virtual reality-augmented rehabilitation for patients following stroke. PhysTher. 2002;82(9):898-915.

[34] Saposnik G, Levin M, Stroke Outcome Research Canada (SORCan) Working Group. Virtual reality in stroke rehabilitation: a meta-analysis and implications for clinicians. Stroke. $2011 ; 42(5): 1380-6$. 
[35] Piron L, Turolla A, Agostini M, Zucconi C, Cortese $F$, Zampolini $M$, Zannini $M$, Dam $M$, Ventura $L$, Battauz M, Tonin P. Exercises for paretic upper limb after stroke: a combined virtual-reality and telemedicine approach. J Rehabil Med. 2009;41 (12):1016-20.

[36] Soares AV, Woellner SS, Andrade CD, Mesadri TJ, Bruckheimer AD, Hounsell MD. The use of Virtual Reality for upper limb rehabilitation of hemiparetic Stroke patients. FisioterapiaemMovimento. 2014;27(3):309-17.

[37] da Silva Ribeiro NM, Ferraz DD, Pedreira É, Pinheiro Í, da Silva Pinto AC, Neto MG, dos Santos LR, Pozzato MG, Pinho RS, Masruha MR. Virtual rehabilitation via Nintendo $\mathrm{Wii} \AA$ and conventional physical therapy effectively treat post-stroke hemiparetic patients. Topics in stroke rehabilitation. 2015;22(4):299-305.

[38] Mouawad MR, Doust CG, Max MD, McNulty PA. Wii-based movement therapy to promote improved upper extremity function post-stroke: a pilot study. J Rehabil Med. 201 1;43(6):527-33.

[39] Hondori HM, Khademi M, McKenzie A, Dodakian L, Lopes CV, Cramer SC. Abstract T MP43: utility of augmented reality in relation to virtual reality in stroke rehabilitation. Stroke. 2014;45(suppl_1):ATMP43.

Salter KL, Teasell RW, Foley NC, Jutai JW.
[40] Outcome assessment in randomized controlled trials of stroke rehabilitation. Am J Phys Med Rehabil . 2007;86(12):1007-12.

[41] Turolla A, Dam M, Ventura L, Tonin P, Agostini M, Zucconi C, Kiper P, Cagnin A, Piron L. Virtual reality for the rehabilitation of the upper limb motor function after stroke: a prospective controlled trial. J NeuroengRehabil. 2013;10(1):85.

[42] Atkins, Jonah. Bridging the gap between reality and virtuality: An analysis of VR technology adoption within architectural practices in the SW. 2017.

[43] Saposnik G, Levin M, Stroke Outcome Research Canada (SORCan) Working Group. Virtual reality in stroke rehabilitation: a meta-analysis and implications for clinicians. Stroke. $2011 ; 42(5): 1380-6$

[44] Laver KE, Lange B, George S, Deutsch JE, Saposnik G, Crotty M. Virtual reality for stroke rehabilitation. Cochrane Database Syst Rev. 2017(11).

[45] Rubio Ballester B. Virtual reality for stroke: is gaming effective for recovery? [Internet]. BMC Blog Network: On Medicine. 2019 [cited 11 June 2019]. Available from: https://blogs.biomedcentral.com/onmedicine/2019/03/07/virtualreality-stroke-gaming-e\%EF\%AC\%80ective-reco very/ 